\title{
EVOLUTION OF CHROMOSPHERIC FINE STRUCTURES ON THE DISK
}

\author{
KATSUO TANAKA \\ Tokyo Astronomical Observatory, Mitaka, Tokyo, Japan
}

\begin{abstract}
Evolution of chromospheric fine structures is discussed from observational points of view. First, various lifetime determinations are reviewed. Then chromospheric oscillation in the interior of supergranule cell is discussed in relation to chromospheric 'grain' structure. We show that the grains are collectively associated with the 180-s oscillations as well as that in $\mathrm{K}$ line the grain is identified with local disturbance generated at lower level and propagating to upper levels. Finally the details of evolution of individual mottles are presented. We show some cases which indicate the propagation of a disturbance along the mottle axis. Statistics of evolution patterns of mottles implies a significant tendency for a system of double dark mottles encasing a bright mottle.
\end{abstract}

\section{Introduction}

Chromospheric fine structure is different between the supergranule cell and its boundary. In the supergranule cell there are many tiny, roundish features called grains. At the boundaries there are several clusters of elongated mottles as well as small granular features called bright mottles or plagette at the central portion of the cluster. Mottles often extend over the cell interior. Morphological relationship between plagette and mottles is similar to the relation between plage and fibrils in active region. Variety of the fine structure might originate from the difference in strength and orientation of the magnetic field. Studies on the evolution have revealed the difference in the evolution between various fine structures. Present knowledge indicates the dominance of oscillatory changes in the cell interior and in plagette as well as in plage; while mottles and fibrils show non-oscillatory changes. Generally evolution of fine structures in the chromosphere would be closely related to the individual process of heat and mass transfers from photosphere to corona. We may evaluate various modes of heat and mass transfers by studying details of the evolution of individual chromospheric features.

In this paper I first review our knowledge of the lifetimes of various features. Then I discuss chromospheric oscillations associated with the grains in the network cell. Finally I try to discuss the details of evolution of individual mottles.

\section{Lifetimes}

Lifetimes have been determined most frequently by a method of survival curve. In this method a group of features to be examined are identified at a certain time first and in good quality frames preceding and following this time numbers of mottles that can be identified with the original ones are counted and plotted against time (survival curve). The full width at half maximum of the survival curve might be de- 
fined as a lifetime. In Table I those lifetimes obtained by this method are shown with an asterisk mark. Some investigators determined lifetimes by following individual features from their birth to disappearance (Bhavilai, 1965, 1966; Bray, 1968, 1969; Sawyer, 1972). The two methods were compared by Sawyer for the same data. She found for the features showing rapid changes faster than the time resolution of the

TABLE I

Lifetimes

\begin{tabular}{|c|c|c|c|}
\hline \multicolumn{4}{|l|}{ Dark fine mottle } \\
\hline de Jager (1957)* & $16 \mathrm{~min}$ & $\mathbf{H} \alpha$ & $0.0 \AA$ \\
\hline Bruzek (1959)* & 13 & & 0.0 \\
\hline Beckers (1964)* & 18 & & \pm 0.5 \\
\hline Howard and Harvey $(1964)^{*}$ & $9 ; 15$ & & 0.0 \\
\hline Macris and Alissandrakis (1970)* & 5.5 & & -0.6 \\
\hline Sawyer $(1972)^{*}$ & 14 & & +0.3 \\
\hline Beckers (1963) & 15.7 & & \pm 0.5 \\
\hline Bhavilai (1966) & 8 & & +0.75 \\
\hline Bray (1968) & 5 & & \pm 0.3 \\
\hline Rogers $(1970)^{* *}$ & 7 & & +0.65 \\
\hline Bhatnagar and Tanaka (1972) & 10 & & -0.5 \\
\hline \multicolumn{4}{|l|}{ Bright fine mottle } \\
\hline Macris and Alissandrakis (1970)* & $12 \mathrm{~min}$ & & $-0.6 \AA$ \\
\hline Sawyer $(1972)^{*}$ & 13 & & +0.3 \\
\hline Bray (1969) & 10 & & \pm 0.3 \\
\hline \multicolumn{4}{|l|}{ Grain } \\
\hline Beckers (1964)* & $4 \mathrm{~min}$ & $\mathrm{~K} 2$ & \\
\hline Sawyer (1972) & 2.2 & $\mathrm{H} \alpha$ & \\
\hline Bhavilai (1966) & 1.3 & $\mathrm{H} \alpha$ & \\
\hline \multicolumn{4}{|l|}{ Fibril } \\
\hline Lippincott (1955) & $-45 \mathrm{~min}$ & & \\
\hline Foukal (1971) & $1-20$ & $\mathbf{H} \alpha$ & \\
\hline \multicolumn{4}{|l|}{ Supergranule network } \\
\hline Macris $(1956)^{*}$ & $26 \mathrm{~h}$ & $\mathbf{K}$ & \\
\hline Janssens $(1970)^{*}$ & 21 & $\mathrm{H} \alpha$ & $+0.65 \AA$ \\
\hline Simon and Leighton $(1964)^{* *}$ & $19-21$ & $\mathbf{K}$ & \\
\hline Rogers $(1970)^{* *}$ & $25.0 \pm 1.6$ & $H \alpha$ & $+0.65 \AA$ \\
\hline
\end{tabular}

* survival curve method (see text).

** cross correlation method.

data that the survival curve gives a misleading result. In the following I briefly discuss the results.

Dark fine mottle. Large spread of lifetime from $5 \mathrm{~min}$ to $18 \mathrm{~min}$ exists in various measurements. There is a slight tendency that a shorter lifetime is obtained for the 
shorter effective time resolution of the data (Sawyer, 1972). It indicates the existence of rapid change. Bray (1968) found that most of the mottles exhibit well-marked changes after $2.5 \mathrm{~min}$. On the other hand it is shown by the visibility curve analyses (Beckers, 1963; Bhatnagar and Tanaka, 1972) that the visibility of mottles never drops for a time longer than $10 \mathrm{~min}$.

Bright fine mottle. Most of the bright fine mottles are located near the center of dark mottles cluster, thus at a lower height than dark fine mottles (Beckers, 1968; Bray, 1969; Banos and Macris, 1970). There are also bright elongated mottles similar to the dark fine mottle (Bhavilai, 1965). Bray (1969) and Macris and Alissandrakis (1970) found that the lifetime of the bright fine mottle is larger by a factor two than the lifetime of the dark fine mottle. Sawyer (1972), however, found the same lifetime as the dark fine mottle. Sawyer's statistics shows rapid changes with a time scale of $1 \mathrm{~min}$. At the central area of dark mottles cluster the intensity oscillation with a period of about $300 \mathrm{~s}$ has been detected by Bhatnagar and Tanaka (1972) in $\mathrm{H} \alpha$ $-0.5 \AA$ and by Liu and Sheeley (1972) in $\mathrm{K} 2{ }_{v}$. These authors measured the intensity variations directly from movies of $\mathrm{H} \alpha$ filtergrams and $\mathrm{K} 2$ spectroheliograms, respectively. Probably the bright fine mottles are responsible for these intensity oscillations.

Grain. From Table I one finds the lifetime of the grain considerably shorter than that of the mottles at the network boundaries. Beckers (1964) found that only $30 \%$ of K grains (bright) are recognized after $2.5 \mathrm{~min}$. Similarly, Sawyer (1972) showed that $72 \%$ of $\mathrm{H} \alpha$ grains (dark) disappear within 2 min. Bhavilai (1966) also found the bright $\mathrm{H} \alpha$ grains in the cell whose median lifetime is $76 \mathrm{~s}$. These results are consistent with the intensity oscillation of a 180-s period found in the supergranule cell by Bhatnagar and Tanaka (1972) and Liu (1973).

Fibril. Foukal (1971b) finds no essential difference between the lifetimes of the fibril and the mottle. It is pointed out by Foukal (1971a) that the visibility of the fibril oscillates in a few minutes about a 'mean appearance' defined by a characteristic alignment, mean darkness and mean length which is preserved typically for several hours. The latter time scale is interpreted by him as similar to that required for major rearrangement of the magnetic field structure. It is similar to the time scale of the mottle-producing activity in the quiet region (Bhavilai, 1966).

Supergranule network. Both survival curve method and cross correlation method give the same lifetime of about one day. The data analyzed by Janssens (1970) and by Rogers (1970) cover $62 \mathrm{~h}$ without an interruption. Time history of the cross correlation Rogers made, indicates a rapid drop of the correlation in the initial $10 \mathrm{~min}$, which corresponds to the lifetime of individual mottles, and more gradual fall of the correlation between $7 \mathrm{~min}$ and $4 \mathrm{~h}$. Rogers interprets this stage of gradual fall as the period of readjustment of the coarse dark mottles. It may be suggested that change in magnetic field structure occurs in this time scale. 


\section{Chromospheric Oscillation in the Network Cell}

It has been known from power spectrum analyses that higher frequencies appear in the 5-min oscillations in the chromosphere. Leighton (1963) and Orral (1966) found the broad peak down to $170 \mathrm{~s}$ in the power spectrum of $\mathrm{K}$ line. Similar results were obtained for $\mathrm{H} \alpha$ by Elliot (1969) and for $\mathrm{H} \beta$ by Bhattacharyya (1972). Recently twodimensional studies have been started by using filtergrams and spectroheliograms. Thus in the central portion of the network cell Bhatnagar and Tanaka (1972) found a well-defined 170 -s period for the intensity oscillation in $\mathrm{H} \alpha-0.5 \AA$. The broad peak which appeared in power spectra in one-dimensional analyses might be due to the mixing of two characteristic periods, namely $170 \mathrm{~s}$ in the interior of the cell and $300 \mathrm{~s}$ at the network boundaries as well as due to th: non-oscillatory components of mottles.

The oscillation is easily detected in time-lapse filtergrams of $\mathrm{H} \alpha$ off-band at which overlying elongated mottles are not so visible. One can see the whole area of the interior of the cell oscillate at any time like the waves in the ocean. Obviously the intensity oscillation in $\mathrm{H} \alpha$ wings is accompanied by discrete dark features called grains. However the size of the oscillatory wave is much larger than the size of individual grain (about $1000 \mathrm{~km}$ ), and occasionally extends over $10000 \mathrm{~km}$. Time-lapse pictures show that the grains tend to appear as a group which takes a form like a chain with a typical length of about $5000 \mathrm{~km}$.

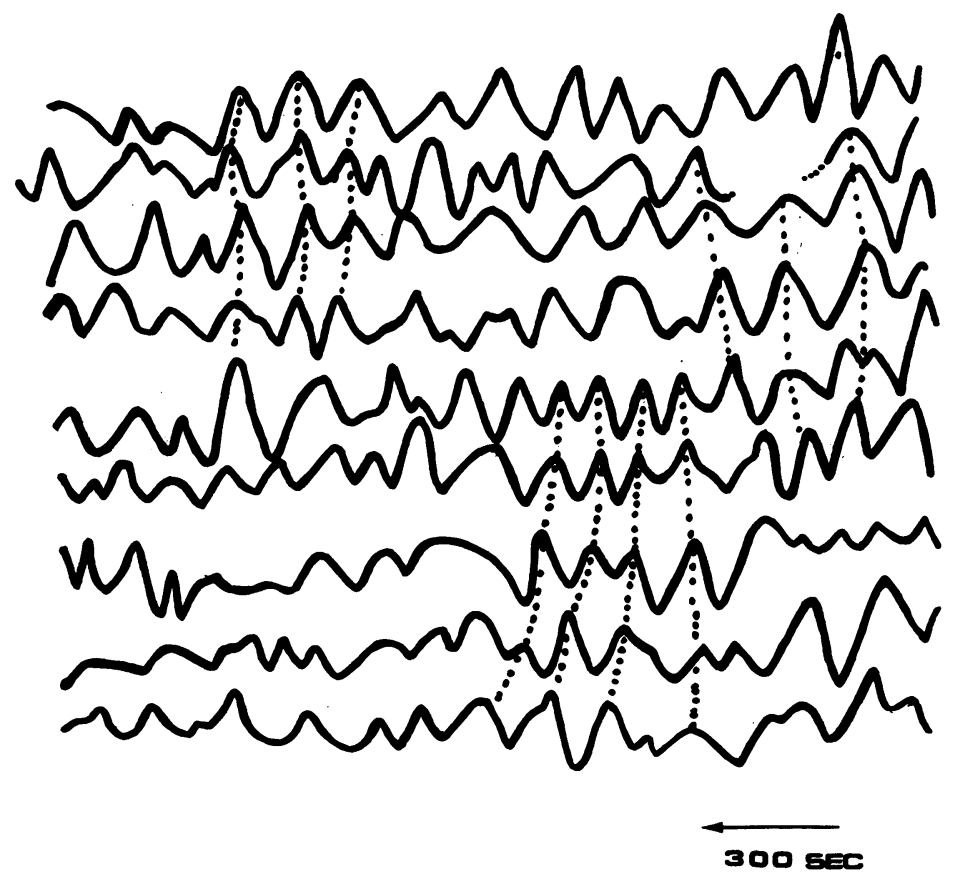

Fig. 1. Sixty-minute records of intensity variation in $\mathrm{K}$ filtergram (bandpass $0.3 \AA$ ) made at the twodimensional points in the cell. Dot lines connect the common peaks of oscillatory waves. Each curve corresponds to one place of $5000 \mathrm{~km}$ diameter. 
In $\mathrm{K}$ filtergrams of bandpass $0.3-0.6 \AA$ the oscillations are more evidently detected since no obscuring materials like mottles exist in such wide bandpasses. Besides the bright $\mathrm{K}$ grains there seem to be groups of dark points which may correspond to the dark phase of the oscillatory change of the $\mathrm{K}$ grain. In Figure 1 intensity oscillations in $\mathrm{K}$ filtergram (Bandpass $0.3 \AA$ ) are shown for two-dimensional points in the interior of the cell. Each curve represents intensity tracing with time at one $5000 \mathrm{~km}$ region. Following records of neighbour points in the ordinate axis one may notice common peaks composing one wave. Time lag between these peaks may lead to apparent horizontal phase velocity of about $100 \mathrm{~km} \mathrm{~s}^{-1}$ within one wave. However, it may not imply actual transverse propagation, but probably represents the difference of arrival time of wavefront at the fixed height of $\mathrm{K}$-line formation. This could happen when the wavefront is originally spherical or when it is modified from plane wave by the effect of magnetic field.

It is interesting to see how the oscillatory wave in the cell interacts with the overlying elongated mottles, whose one end originates at the cell boundary. I have found a few cases in which the grains are formed along the mottle axis as the mottle stretches to the cell interior. They suggest that the top of mottles and grains are not independent phenomena; however their relation on the whole has not been clarified yet.

From a theoretical point of view an attempt has been made to interpret the 180 -s oscillation as a trapped oscillation in the chromosphere where the horizontal magnetic fields prevail (Nakagawa, 1973). In such a model, the chromosphere is considered as a continuous medium; discrete features like grains are then secondary phenomena such as local excitation or condensation due to the oscillation. On the other hand there is phenomenological evidence to identify the grain itself as individual propagation of locally confined waves as will be discussed next.

\section{Evolution of $\mathrm{K}$ and $\mathrm{H} \alpha$ Line Profiles in the Network Cell}

Formation of $\mathrm{K}$-line profile, especially the origin of its asymmetry is a still controversial problem. Recently a new aspect has appeared in this problem, namely evolutionary changes of K-line profiles. Wilson and Evans (1971) and Wilson et al. (1972) have found various patterns of the evolution as follows:

(1) a single-peak emission evolves into a double-peak emission;

(2) a double-peak emission evolves into a single-peak emission at the blue or red wings;

(3) a double-peak emission with a red (blue) asymmetry changes to a blue (red) asymmetrical emission.

These changes are found to occur with a time scale between 10 and $60 \mathrm{~s}$ mainly in the central portion of the cell.

The evolution of $\mathrm{K}$-line profiles in the cell has been studied extensively by Liu $(1972,1973)$. He has found in several places, not large in number, that brightening moves progressively from the very far wing of $\mathrm{K}$ line $( \pm 7 \AA)$ towards the line core up to K2 (Figure 2). As the brightening propagates the profile of $\mathrm{K}$-line changes from 
pure absorption profiles with enhanced $\mathrm{K} 1$ wing to intense single-peak emission at $\mathrm{K} 2_{v}$. The whole phenomenon occurs within about $100 \mathrm{~s}$ and tends to repeat with a period of $180 \mathrm{~s}$. This could be considered as a particularly bright grain.

Most of the places (grains) inside the cell Liu (1973) studied show intensity oscillation with the same period as the above phenomenon, but with relatively small time

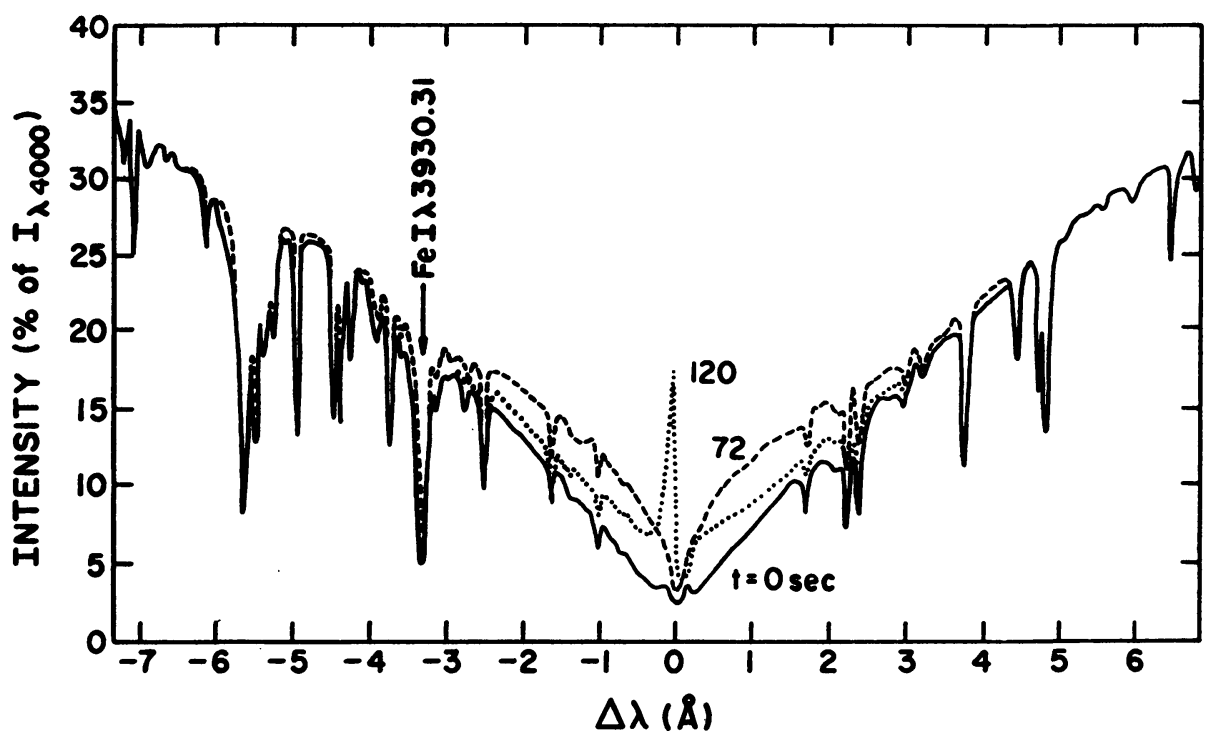

Fig. 2. Evolution of the K-line profile of the grain which shows particularly large phase lags between various wavelengths. At $t=0$, the undisturbed profile. At $t=72 \mathrm{~s}$ the disturbance has propagated from the far wing to the inner wing. At $t=120 \mathrm{~s}$ an intense $\mathrm{K}_{v}$, peak and the wing starts to recover.

(Courtesy of S. Y. Liu).

lags between various wavelengths. One such example is shown in Figure 3 which exhibits intensity tracings at 7 different wavelengths. One can see conspicuous intensity oscillation with a 180-s period except $\mathrm{K} 3$ core where the amplitude of oscillation is reduced. There are phase lags between wavelengths in a sense that the brightening in the wing precedes that near the core. Liu found that $97 \%$ of all the 459 peaks show this tendency. The mean time lag between bright peaks at K1 wing $( \pm 0.44 \AA)$ and $\mathrm{K} 2{ }_{v}$ is found equal to $9.3 \mathrm{~s}$. This value is considerably smaller than the mean period of $180 \mathrm{~s}$. If we consider also that the intensity enhancements are symmetrical in both wings beyond $\mathrm{K} 2$, it could be concluded that the 180 -s oscillation in $\mathrm{K}$ line wing is a pure brightness oscillation.

Liu (1973) interprets these phenomena as the propagation of wave from below the temperature minimum, assuming that the intensity fluctuations are due to actual enhancement of temperature in an optically deep region. For this explanation he assumes the dissipation due to acoustic wave; if one assumes a height difference of $500 \mathrm{~km}$ between the levels forming $\mathrm{K} 2_{v}$ and $\mathrm{K} 1( \pm 0.44 \AA)$, and takes into account the mean time lag $9.3 \mathrm{~s}$, one finds a vertical phase velocity $v_{\mathrm{p}}=54 \mathrm{~km} \mathrm{~s}^{-1}$ and a group 
velocity $v_{\mathrm{g}}=0.67 \mathrm{~km} \mathrm{~s}^{-1}$ from the relation $v_{\mathrm{p}} \cdot v_{\mathrm{g}}=c^{2}$ with $c=6 \mathrm{~km} \mathrm{~s}^{-1}$. Finally using velocity amplitude $\left(1.5 \mathrm{~km} \mathrm{~s}^{-1}\right)$ obtained from the strong line near $\mathrm{K}$-line center Liu finds mean mechanical flux in the grain equal to $2 \times 10^{7} \mathrm{erg} \mathrm{cm}^{-2} \mathrm{~s}^{-1}$.

Some of this flux is radiated in $\mathrm{K}$ line itself; subtracting the flux radiated in $\mathrm{K}$ line a net mechanical flux of $7 \times 10^{6} \mathrm{erg} \mathrm{cm}^{-2} \mathrm{~s}^{-1}$ is obtained. This value would be re-

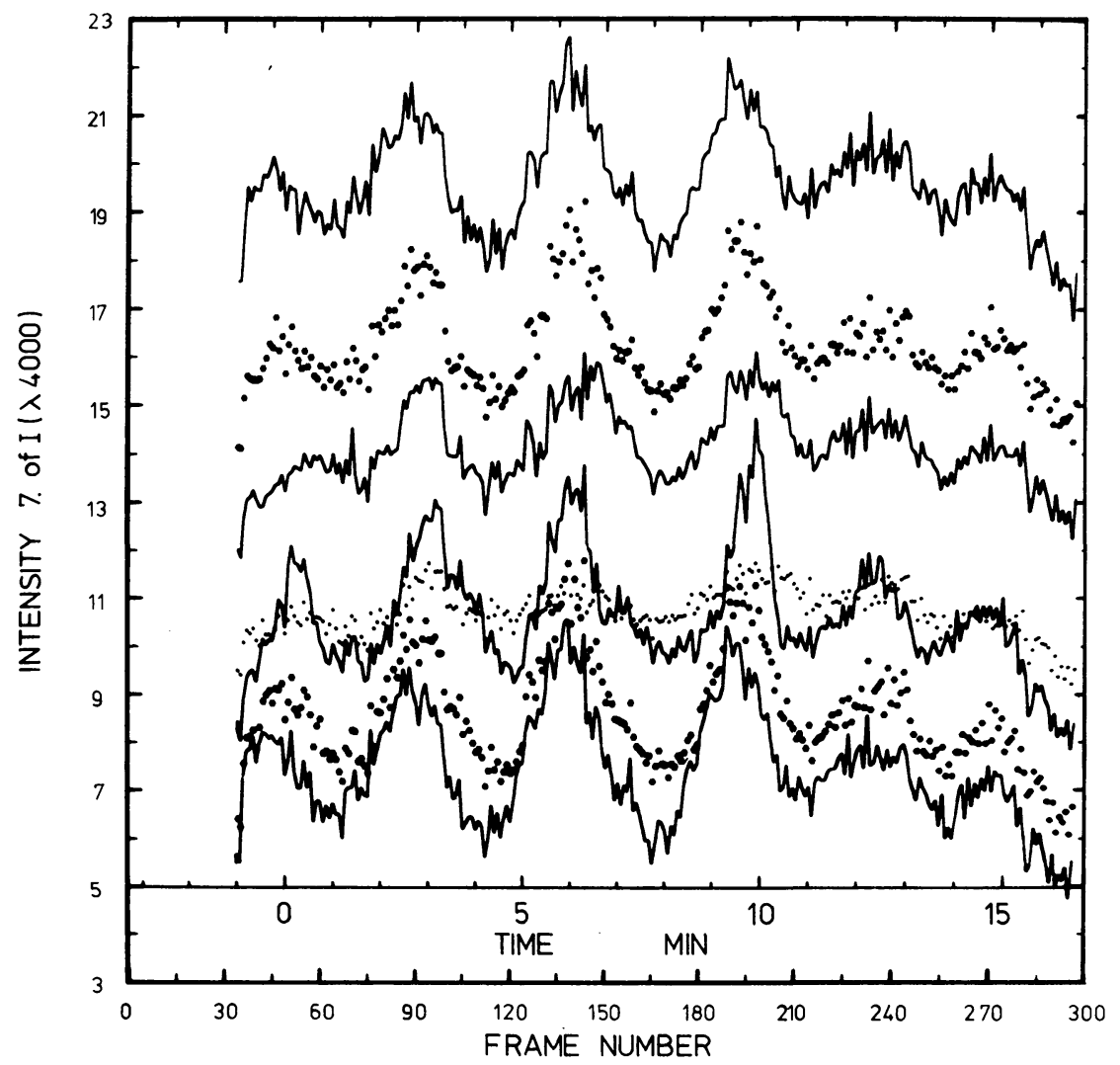

Fig. 3. Example of the intensity oscillation of the grain at various wavelengths (from top to bottom: $-0.44 \AA, \mathrm{K} 1_{v}, \mathrm{~K} 2_{v}, \mathrm{~K} 3, \mathrm{~K} 2_{r}, \mathrm{~K} 1_{r},+0.44 \AA$ ). Note the phase coherency at various parts of profile. (Courtesy of S. Y. Liu).

duced if we consider the fractional area of grains and radiation loss from other lines. In conclusion, as far as we adopt the hypothesis of acoustic wave, the flux carried by grains seems to be insufficient for the coronal heating, which requires $5 \times 10^{6} \mathrm{erg}$ $\mathrm{cm}^{-2} \mathrm{~s}^{-1}$ on the whole surface of the Sun.

At the various phases of oscillation the grain exhibits various $\mathrm{K}$-line profiles. Statistics shows that $50 \%$ have double-peak emissions, $30 \%$ have single-peak emissions and $20 \%$ have pure absorption profiles (Liu and Smith, 1971). In Pasachoff's (1970) result there are more single-peak emissions although he did not specify the position. The single-peak emission appears most frequently at the phase when the bright grain appears at K2, or when the disturbance reaches the highest level. The pure absorption 
profile corresponds to the dark phase of the grain or the time when the disturbance is generated at lower level. It is to be noted that the single-peak emission at $\mathrm{K} 3$ is not observed generally.

The time variation of $\mathrm{H} \alpha$ line profile is qualitatively different from $\mathrm{K}$ line. Figure 4 shows the intensity tracings with time at three wavelengths of $\mathrm{H} \alpha(0 \AA, \pm 0.5 \AA)$; the phase differs by about $180 \mathrm{deg}$ in the two wings implying velocity oscillation contrary to the brightness oscillation in $\mathrm{K}$ line. We may note also that this velocity oscillation

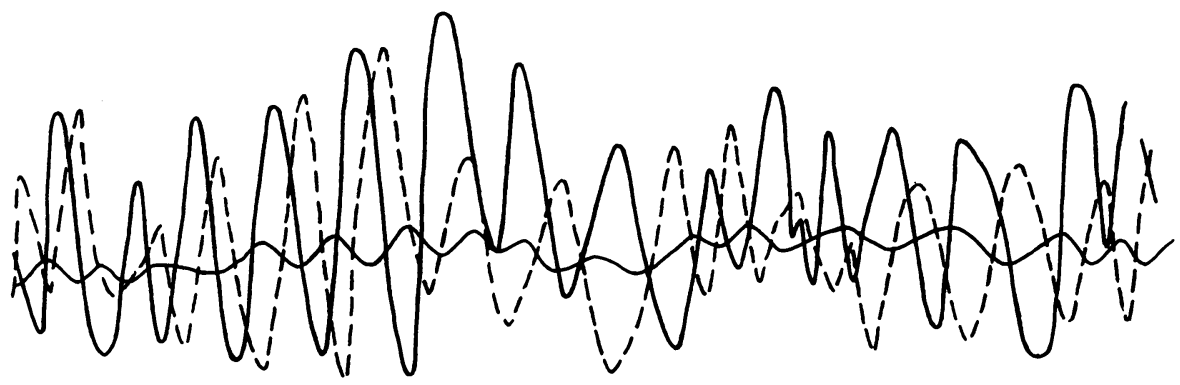

Fig. 4. Intensity tracings of time-lapse $\mathrm{H} \alpha$ spectrograms made at the central portion of the cell. Time proceeds from right to left. Note the phase difference between $-0.5 \AA$ (thick line) and $+0.5 \AA$ (broken line). The mean interval between intensity peaks is $180 \mathrm{~s}$.

is caused by the Doppler shifts of $3-5 \mathrm{~km} \mathrm{~s}^{-1}$ in the individual dark features, namely grains. Since this amplitude is well within the width of $\mathrm{K} 3$ core, the velocity oscillation apparently missing in $\mathrm{K}$ line may well be confined in $\mathrm{K} 3$ core, where the brightness oscillation is reduced. It might be suggested that two kinds of oscillations exist always associated with the grain phenomenon: the brightness change originates at lower level and propagates upwards and the velocity perturbation is produced at higher levels, which would be seen in $\mathrm{H} \alpha$ and $\mathrm{K} 3$.

While the relation between the two kinds of oscillations remain uncertain, the interpretation of $\mathrm{K}$-line profiles due to two vertical components (two clouds) looks interesting. In this model the dark feature absorbs various parts of $\mathrm{K} 3$ core of the relatively wide emission originated at slightly lower level than the dark feature; thus double or single-peak profile can be produced as the phase of the velocity oscillation of the dark feature changes. Independent of this dynamical model Suemoto (1971) has examined the detailed characteristics of $\mathbf{H}$ and $\mathrm{K}$ lines profiles by two vertical components model taking their velocity distributions into account and succeeded in fitting the observed mean and rms profiles by adjusting optical thicknesses of the two components and number of elements superposed in the line of sight. The optical thicknesses in $\mathrm{K}$ line center equal to 1000 and 10 are obtained for the bright feature and the upperlying absorption feature respectively.

Another interpretation of time-dependent $\mathrm{K}$-line profiles has been proposed by Cram (1973), who considers the height variation of source function in a single structure. In this case transfer equation in presence of velocity field would represent the problem. 
One characteristic feature in the $\mathrm{H} \alpha$ spectra of the grains is the tilt of profile to the direction of dispersion. There has been observed a reversal of this tilt against the dispersion direction in a time of about $1 \mathrm{~min}$. For the tilt of emission line observed outside the limb Beckers (1972) suggested two explanations: (1) merging of two or more unresolved features; (2) rotation or differential motion in the structure. In case of the grain the tilt is evidently associated with the velocity oscillation of two neighbouring features in favor of the first explanation.

\section{Evolution of Individual Mottles at the Network Boundary}

Evolution of elongated mottles located at the supergranule boundaries are very complex since not only brightness but size and shape changes continuously. Howard and Harvey (1964) called these changes seething motion. When we look at the intensity variation of one dark mottle, continuous fluctuations are found with a time scale from $1 \mathrm{~min}$ to $5 \mathrm{~min}$. Most of them are by no means periodic. The basic problem in the evolution of mottles would be to determine the fundamental mode of these changes. Particularly in relation to spicule it would be important to know the direction of the fundamental motions, whether it occurs along the axis of mottle or perpendicular to it.

Beckers $(1963,1964)$ studied time variations of mottles visibilities in the blue and red wings $( \pm 0.5 \AA)$ of $H \alpha$. Out of 112 mottles he finds $41 \%$ show the visibility maximum first in the blue wing and then in the red wing with a mean time lag of $7.0 \mathrm{~min}$, $35 \%$ show periodic visibilities and $24 \%$ show no distinct variation during $20 \mathrm{~min}$. Beckers interprets the mottles in the first type as rising and falling spicules. Generally the velocity determination by using filtergrams is not straightforward (Athay, 1970). However, so far as a mottle can be considered as a cloud isolated from any other medium as has been shown by Grossmann-Doerth and von Uexküll (1971), it would be correct to interpret a blue sentive mottle as rising.

Bhavilai (1965) and Dunn (1971) have shown that some mottles are visible only in blue wing, while others appear only in red wing. In a cluster of mottles the positions of blue and red mottles are mixed. Bhavilai (1965) considered a flow of material in a loop to explain this feature. We have no clear evidence at present whether the mottles occur in an open field or in a closed field such as the loop although faint connection is occasionally seen between two centers of the clusters of mottles suggesting the loop structure (see Figure 7(e), (i) for example).

Rising and falling velocities as inferred from $\mathrm{H} \alpha$ spectra of mottles are considerably smaller $\left(4 \mathrm{~km} \mathrm{~s}^{-1}\right)$ compared to the rms velocity of the spicule $\left(15 \mathrm{~km} \mathrm{~s}^{-1}\right.$ according to Pasachoff et al., 1968). Grossmann-Doerth and von Uexküll (1973) attempted to explain this discrepancy due to the decrease of contrast by seeing; it is found that the observed velocity is reduced to $10-25 \%$ of the true velocity provided that the size of mottle is about the same as the spatial resolution. Under the ideal seeing condition we might expect $15-40 \mathrm{~km} \mathrm{~s}^{-1}$ for the Doppler shift on the disk.

Direct evidence of upward motion of mottles can be obtained by examining evo- 
lution of an individual mottle. I have found that the top of the mottle extends with an apparent velocity from $20 \mathrm{~km} \mathrm{~s}^{-1}$ to $200 \mathrm{~km} \mathrm{~s}^{-1}$. The mean velocity is $55 \mathrm{~km} \mathrm{~s}^{-1}$. The frequency of elongation at one fixed position is $0.17 \mathrm{~min}^{-1}$. We can see that a mottle starts to stretch suddenly from its original length or from the center of cluster and continues to stretch for a duration $20 \mathrm{~s}$ to $2 \mathrm{~min}$. Although it indicates some transfer from below it is uncertain as yet whether it is associated with actual mass motion or wave propagation.

Under the high spatial resolution it is common to see a system of the double dark mottles with a bright gap between them. Bhavilai (1965) found this feature which he called double chains. Tanaka (1972) found about $30 \%$ of all the dark mottles are double or can be resolved into double at any one instance. It consists of the two parallel, straight or curved dark mottles with a separation of $1^{\prime \prime}$ or less and a gap between them which is brighter than the background. In $\mathrm{H} \alpha$ center line picture the lower part of the bright gap is often identified with the bright elongated mottle; it loses its contrast in the wing picture, thus only the double mottles are visible there. Figure 5 illustrates double mottles taken at $\mathrm{H} \alpha-0.5 \AA$.

It has been found that any mottle shows a stage of 'double mottles' in its evolution; statistically it occurs at one position in the cluster of mottles every $4.4 \mathrm{~min}$ al-
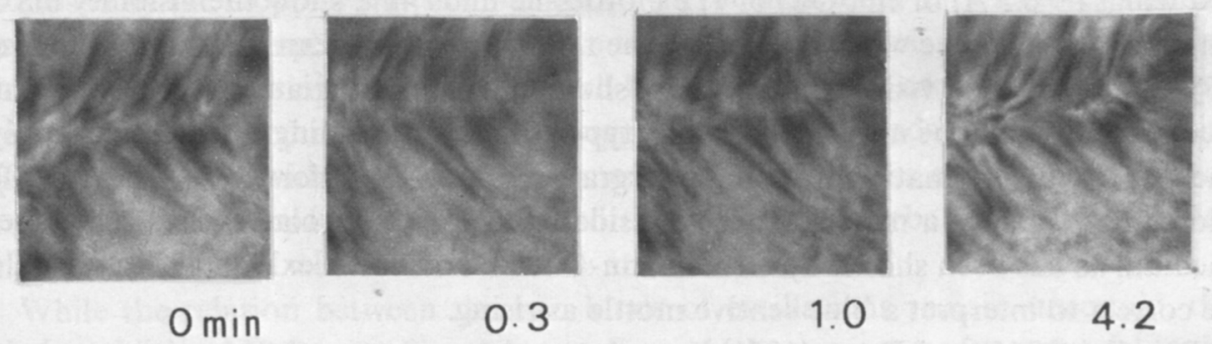

0.3

1.0

4.2

Fig. 5. Example showing the double mottles at $\mathrm{H} \alpha-0.5 \AA$. Note the stretch and shrink with time. One side of each picture corresponds to a scale $25^{\prime \prime}$.

though the occurrence is not always periodic. The lifetime of this system ranges from $10 \mathrm{~s}$ to $15 \mathrm{~min}$ with the average of $40 \mathrm{~s}$. Their evolutions are shown in Figures 6 and 7.

In the final part of this paper I try to classify patterns of the evolution of mottles in the quiet region. For data I used filtergrams taken at $\mathrm{H} \alpha-0.3 \AA$ every $10 \mathrm{~s}$ for 40-min duration. Table II summarizes distribution of numbers of various phases which can be found at the position of a mottle; the numbers are the averages of 70 mottles out of 10 clusters. Phases of a mottle include (1) double mottles system, (2) multistreaks or several faint dark streaks parallel to each other, (3) one or more roundish knots, (4) diffuse cloud-like stage, (5) short dark mottle or a group of short segmentary mottles with various orientations. Among these various phases the double 


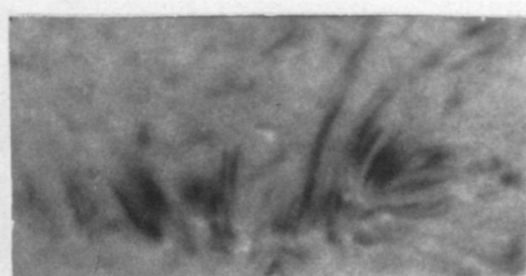

a

$129 \mathrm{sec}$

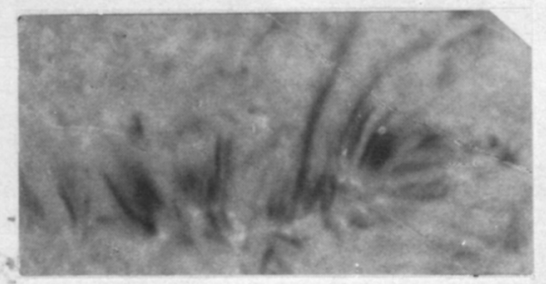

140

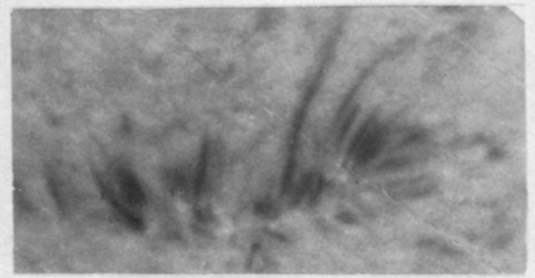

152

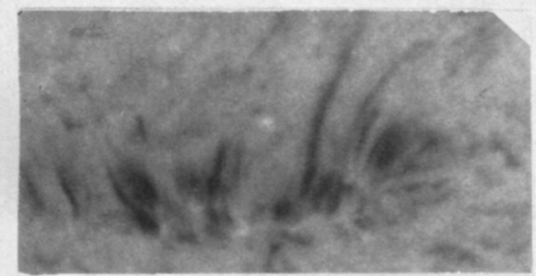

b

164

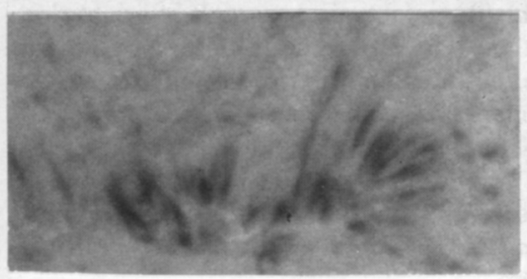

211

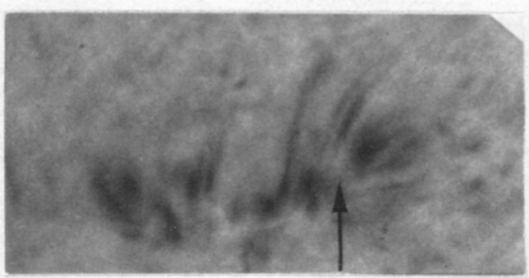

C 234

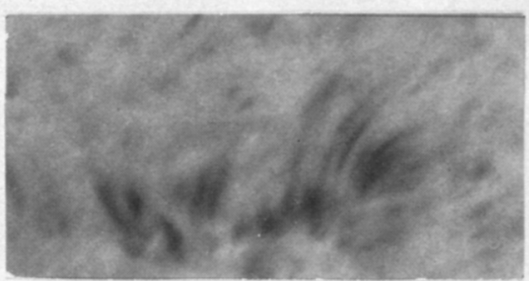

257

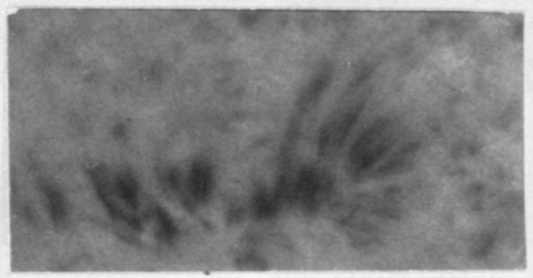

291
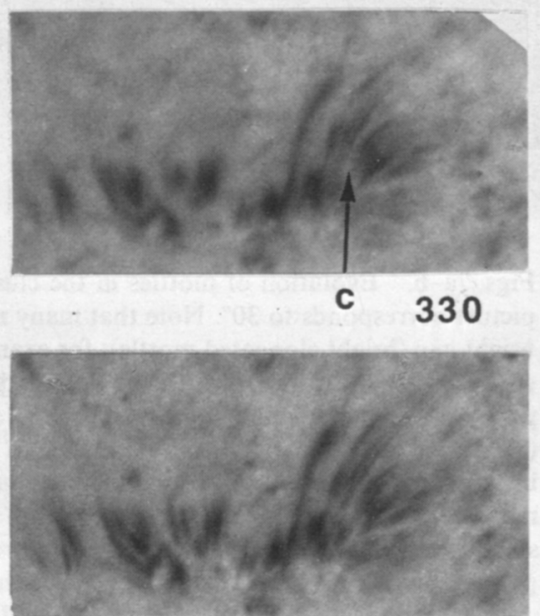

345

Fig. 6. Evolution of mottles in semi-active region $(\mathrm{H} \alpha-0.7 \AA)$. (a) double mottles, (b) widening of the double mottles, (c) the double mottles (234 s) splits into the narrower double mottles ( $330 \mathrm{~s})$. This may be a newly emerging double mottles system. See text for discussion. See many double mottles at the frame $345 \mathrm{~s}$. 


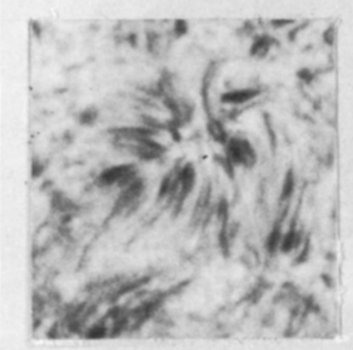

$0 \mathrm{~min}$

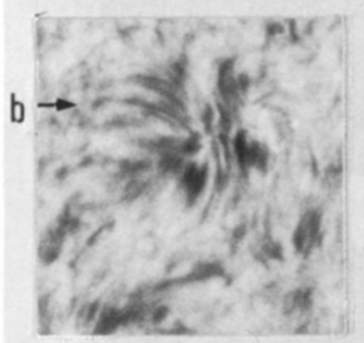

5. 3

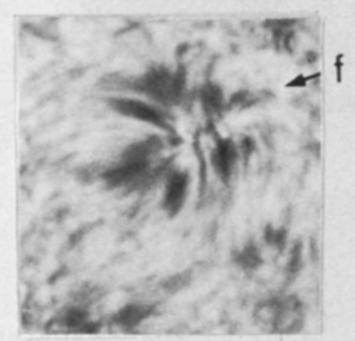

10. 8

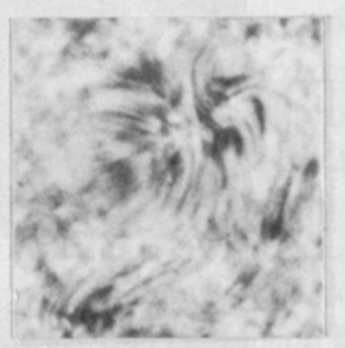

0. 8

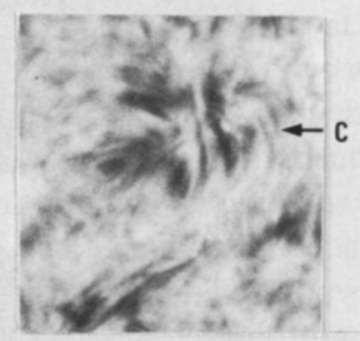

$8 \cdot 8$

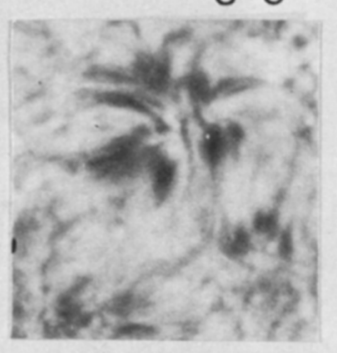

11.0

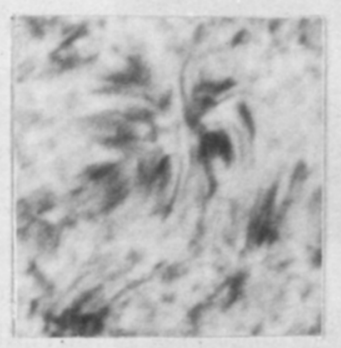

1. 2

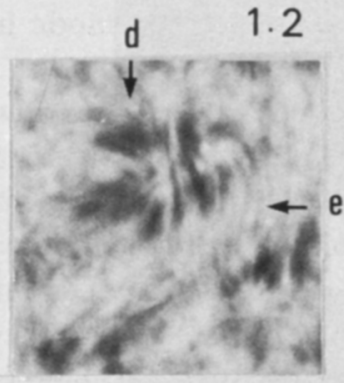

9.2

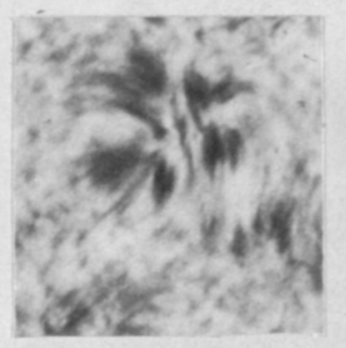

11.8

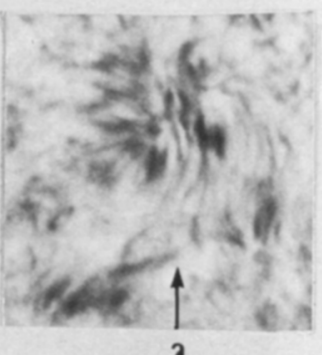

4.8

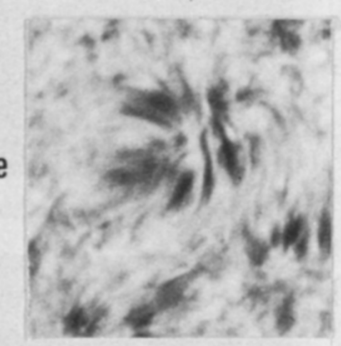

9.5

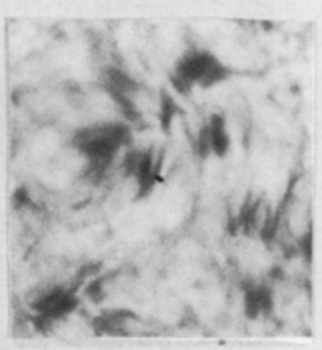

17.8

Fig. 7a.

Figs. 7a-b. Evolution of mottles in the cluster (rosette) in quiet region $(\mathrm{H} \alpha-0.3 \AA)$. One side of each picture corresponds to $30^{\prime \prime}$. Note that many mottles exhibit a system of the double dark mottles with the bright gap (bright elongated mottle); for example (a), (b), (j), (k), (1) etc. (a) typical example of bright elongated mottle stretching through the double dark mottles, (b) three sets of the double mottles, (c) a bright point $(8.8 \mathrm{~min}$ ) elongates slightly $(9.5 \mathrm{~min})$, (d) transfer of the diffuse matter between neighbour mottles ( 8.8 to $9.5 \mathrm{~min}$ ), (e) and (i) faint connection between two centers of the clusters, (f) connection of the broken mottles $(10.8 \mathrm{~min})$ to the curved double mottles $(11.0 \mathrm{~min})$, (g) stretching of the curved double mottle, (h) transient short double mottles, (j) stretching of the double mottles $(20.0 \mathrm{~min})$ from the broken stage of mottles (19.2 $\mathrm{min})$, (k) apparent lateral shift of the double dark mottles (22.5-23.7 min), (1) bright point grows at $25.0 \mathrm{~min}$, extends through the double mottles $(26.3-27.0 \mathrm{~min})$. Note also the stretch of the double dark mottles.

mottles system is the only well-developed stage of a mottle although sometimes the double mottles are hardly resolved. Also it matches with the phase that a mottle stretches and becomes dark. Stages from (2) to (5) occur in general in the intermediate phases between the double mottles stage.

On the whole there do exist some features corresponding to (1) to (5) when one 

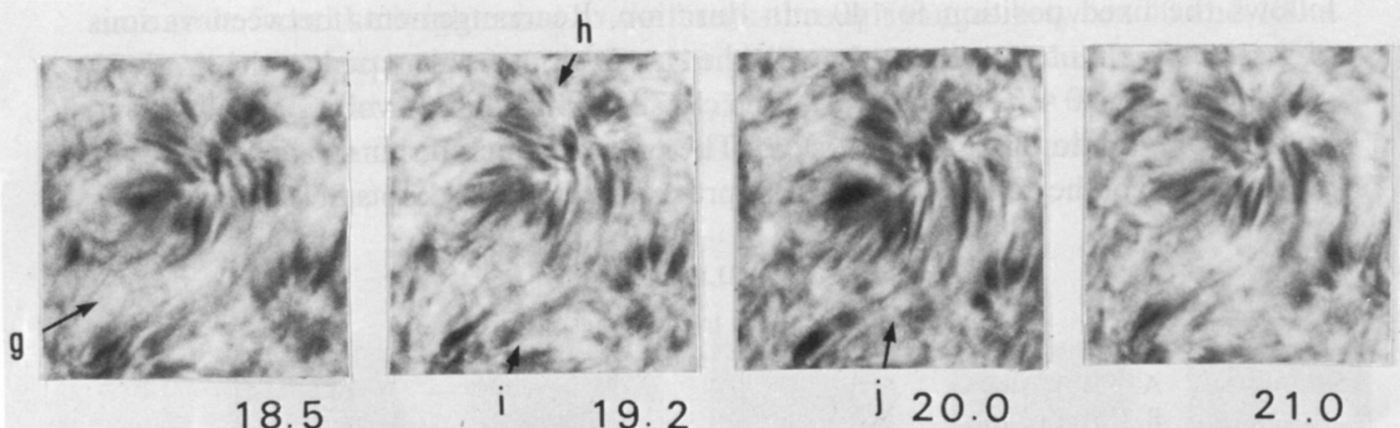

18.5

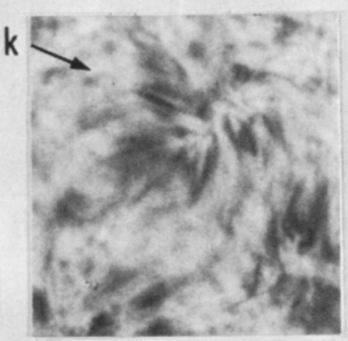

j 20.0

21.0
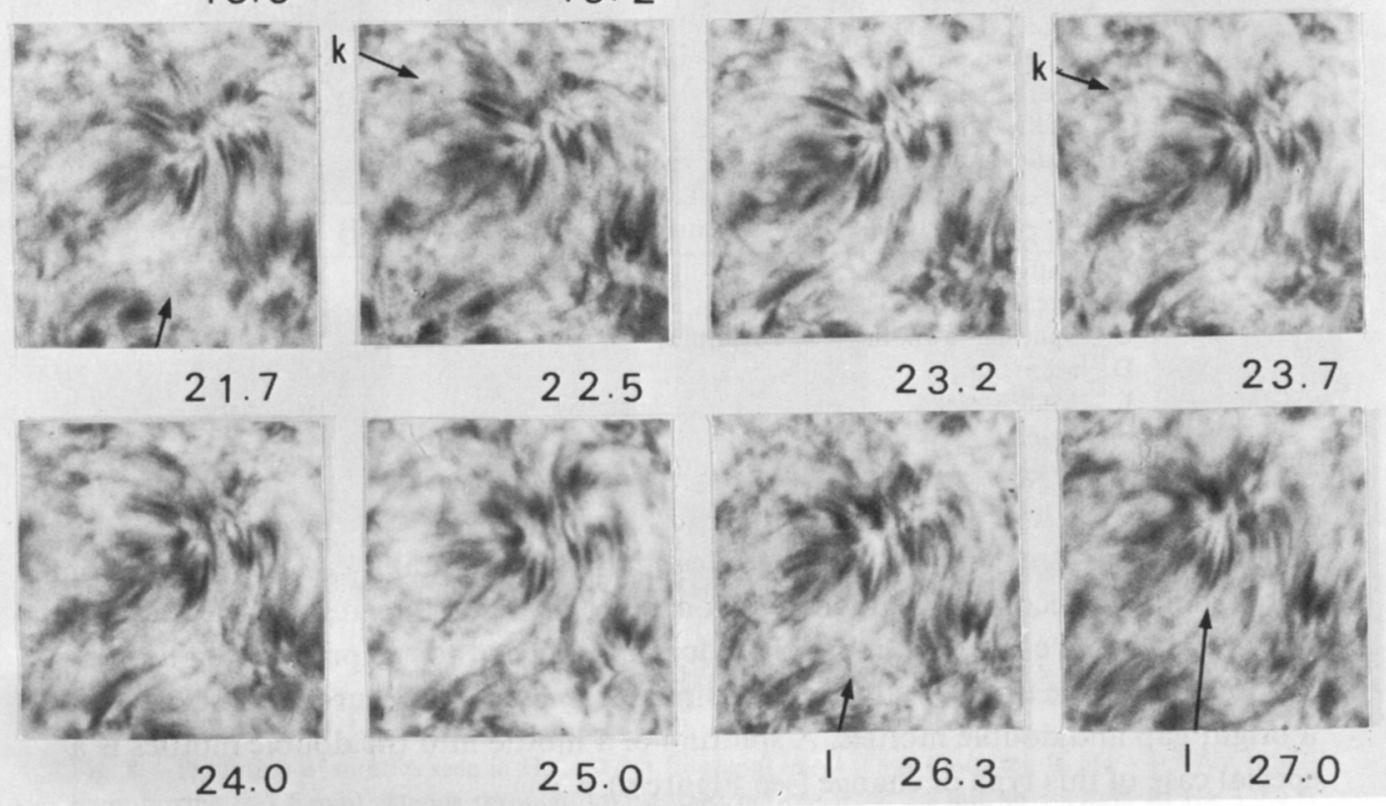

Fig. $7 b$.

TABLE II

Mean frequency of various phases of a mottle at one position during $40 \mathrm{~min}$

\begin{tabular}{|c|c|c|c|c|c|}
\hline & Double system & Multi-streaks & Knots & Diffuse & Short \\
\hline Quiet & 9.8 & 1.7 & 1.4 & 4.4 & 3.2 \\
\hline \multirow[t]{2}{*}{ Semi-active } & 21.9 & 5.7 & 3.2 & 7.2 & 7.7 \\
\hline & Slow stretch ${ }^{a}$ & Darkening & & & \\
\hline Quiet & 6.2 & 3.1 & & & \\
\hline Semi-active & 7.3 & 3.8 & & & \\
\hline
\end{tabular}

a Visually recognizable stretch with velocities smaller than $500 \mathrm{~km} \mathrm{~s}^{-1}$. See text for the definitions of various phases. 
follows the fixed position for 40-min duration. Rearrangements between various phases occur almost continuously with the shortest time scale equal to the time resolution namely $10 \mathrm{~s}$. Table III shows the classifications of the evolutionary patterns to and from the double mottles system. The pattern which occur most frequently is the formation of the double mottles from pre-existing streaks, knots or small segments

TABLE III

Phases before evolving to the elongated mottles

\begin{tabular}{lll}
\hline A. & Multi-streaks & $20.5 \%$ \\
B. & Multi-knots & 18.5 \\
C. & Short scattered mottles & 17 \\
D. & Bright point $\rightarrow$ Bright elongated mottle with double mottles & 15.7 \\
E. & Dark point at the root & 14.5 \\
F. & Diffuse cloud & 9.6 \\
G. & Diffuse knot & 4.8 \\
\hline
\end{tabular}

\begin{tabular}{lll}
\hline \multicolumn{2}{c}{ Phases to which elongated dark mottle evolves } \\
\hline A. & Diffuse cloud & $29 \%$ \\
B. Break-up & 25 \\
C. Splitting to another double mottles & 19 \\
D. Shortening & 11 \\
E. Multi-streaks & 10 \\
F. Knots & 7 \\
\hline
\end{tabular}

(A, B, C, see Figures 6 and 7 for examples); in this case small, scattered mottles or knots suddenly become straightened or connected into the elongated mottles (double mottles). In some cases dark mottles are formed adjacent to the pre-existing mottles as though they are transferred to lateral direction. When this occurs one finds always a bright gap and double mottles. A splitting of a mottle into the double mottles is a special case of this type of change (see Figure 6).

For mechanism of sudden change of mottles visibility we may suggest (a) rapid change of the Doppler shift due to the oscillatory wave, (b) rapid condensation from pre-existing matter, (c) wave propagation along the axis of mottles. The last explanation is analogous with the evolution described below although the velocity of propagation larger than $500 \mathrm{~km} \mathrm{~s}^{-1}$ is required to explain the rapid formation such as restricted by the time resolution.

An important pattern of the evolution is the stretching of the double mottles from the central part of the cluster of mottles (D, E). Type D would be particularly interesting as it suggests some heat transfer from lower chromosphere to corona along the magnetic field line. A bright point appears first at the central portion of the cluster, increases its size and intensity and finally elongates through the double dark mottles, which develop together with the bright mottle elongation (see examples in Figure $7 \mathrm{a}, \mathrm{c}, \mathrm{j}, 1$ and Figure 8). Occasionally one can recognize the top of the bright elongated mottle moving outwards with a velocity $20-40 \mathrm{~km} \mathrm{~s}^{-1}$. Then it loses its contrast near the top of the double dark mottles as if it becomes too hot to be visible in $\mathrm{H} \alpha$. The 
time scale from the brightening to the break-through ranges between 40 and $100 \mathrm{~s}$. In the red wing picture we see a diffuse bright mottle appear at the intermediate position of mottles at $3.5 \mathrm{~min}$ after the break-through occurred in the blue wing (see Figure 8). This amount of the time lag is very common for the morphological similarity between the blue and red wings.

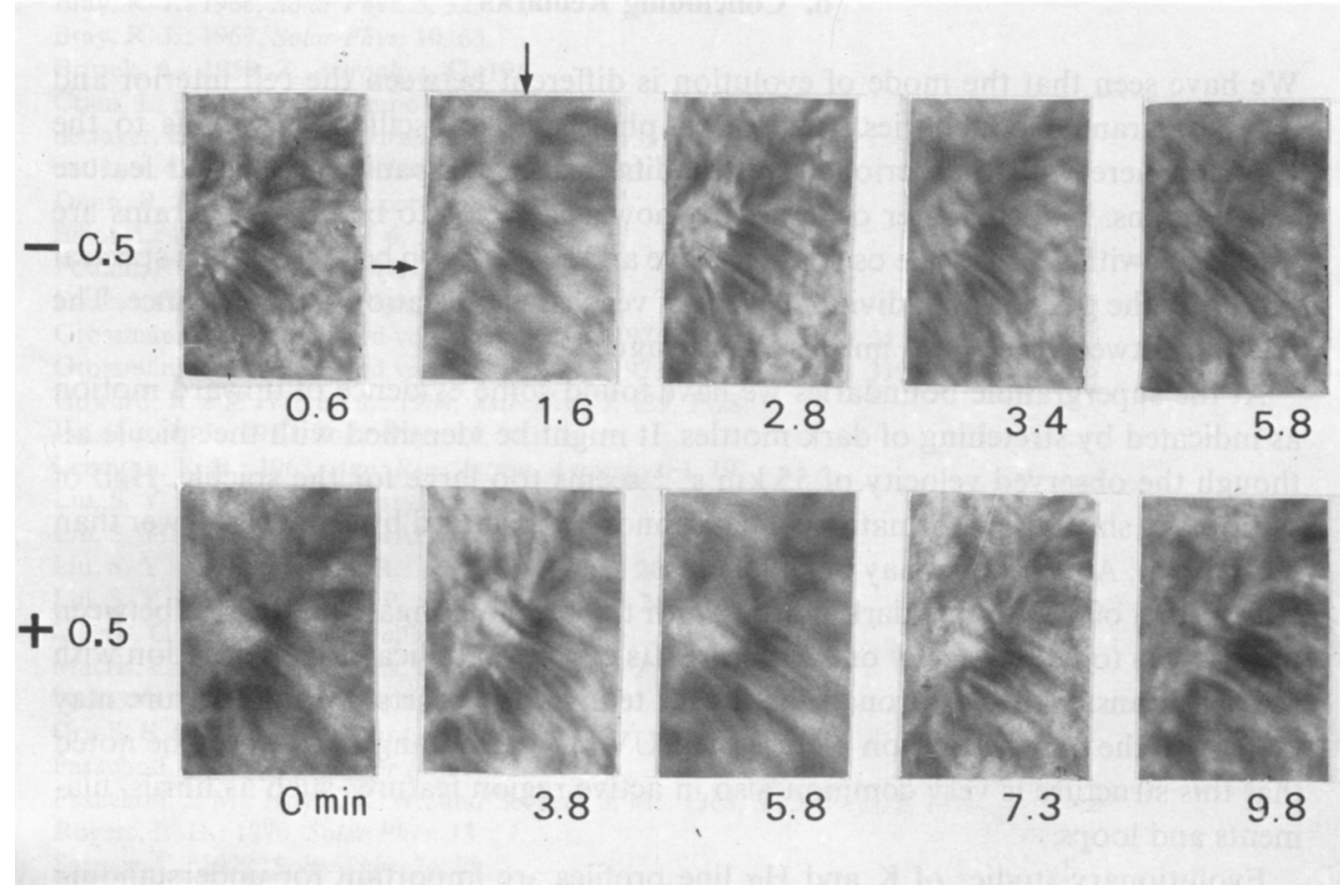

Fig. 8. Evolution of mottles seen in $\mathrm{H} \alpha-0.5 \AA$ (top) and $\mathrm{H} \alpha+0.5 \AA$ (bottom). In $\mathrm{H} \alpha-0.5 \AA$ a bright point (arrow at $1.6 \mathrm{~min}$ ) extends through double dark mottles from $2.8 \mathrm{~min}$ to $3.4 \mathrm{~min}$. In $\mathrm{H} \alpha+0.5 \AA$ bright feature appears at $5.8 \mathrm{~min}$ at intermediate position between the root and top of the diffuse dark mottle at $5.8 \mathrm{~min}$. The double mottles with a bright gap are seen after $7.3 \mathrm{~min}$ and gradually fall towards the root. There is a time lag of $4 \mathrm{~min}$ between the appearances of the double mottles in $\mathrm{H} \alpha-0.5 \AA$ and $+0.5 \AA$.

The outgoing velocity of this phenomenon is similar to the typical velocity of the spicule. We may note that spicule spectra of $\mathrm{H} \alpha$ show the double structures like the double mottles although they appear usually in one wing. It is suggested that this phenomenon or bright mottle elongation through double dark mottles might be considered as a physically meaningful spicule phenomenon. I assume that a bright elongated mottle or bright gap represents a hot core of the spicule and the double mottles are a cold cylindrical envelope or two discrete features formed at the boundaries of the hot core.

Summarizing the evolution of mottles $30 \%$ are formed due to the stretching from shorter features and $50 \%$ are reformed from pre-existing materials. However there 
is no way to reject a possibility that the latter case denotes very fast stretch independent of the pre-existing materials. It is to be added that we often see apparent falling of the dark fine mottles in the movies taken near the limb. But this case has not been detected so often in this analysis made at disk center.

\section{Concluding Remarks}

We have seen that the mode of evolution is different between the cell interior and the supergranule boundaries. In the cell photospheric oscillation extends to the chromosphere with the period being modified. It accompanies individual feature called grains. The character of the grain, however, seems to be dual: (1) grains are associated with a large scale oscillatory wave and show group behavior, (2) in spectral evolution the grain is an individual event of vertical propagation of disturbance. The relation between these two images is still vague.

At the supergranule boundaries we have found some evidence of upward motion as indicated by stretching of dark mottles. It might be identified with the spicule although the observed velocity of $55 \mathrm{~km} \mathrm{~s}^{-1}$ seems too large for the spicule. Half of the mottles show rapid formation which cannot be explained by a velocity lower than $500 \mathrm{~km} \mathrm{~s}^{-1}$. Alfvén wave may be invoked.

A system of the double dark mottles with the bright elongated mottle in between them seems to be physically one structure. Its evolution indicates close relation with the heat transfer to the corona. The higher temperature parts of this structure may be seen in the high resolution pictures of EUV and XUV ranges. It should be noted that this structure is very common also in active region features such as fibrils, filaments and loops.

Evolutionary studies of $\mathrm{K}$ and $\mathrm{H} \alpha$ line profiles are important for understanding the dynamical behaviors of the network cell. Such study is also necessary for the supergranule boundaries.

Studies on the evolution of individual fine structures at various levels would clarify the individual process of the generation of the disturbance in the granule, its propagation and development in the chromosphere and beat transfer to the corona. In particular we need to study the height between the granule and the mottle as well as the transition region between the chromosphere and the corona.

I wish to thank Dr H. Zirin for providing the data for this analysis and Drs Z. Suemoto, T. Hirayama, Y. Uchida, and E. Hiei for valuable discussions.

\section{References}

Athay, R. G.: 1970, Solar Phys. 12, 175.

Banos, G. J. and Macris, C. J.: 1970, Solar Phys. 12, 106.

Beckers, J. M.: 1963, Astrophys. J. 138, 648.

Beckers, J. M.: 1964, Thesis, Utrecht; AFCRL Env. Res. Papers No. 49.

Beckers, J. M.: 1968, Solar Phys. 3, 367. 
Beckers, J. M.: 1972, Ann. Rev. Astron. Astrophys. 10, 73.

Bhatnagar, A. and Tanaka, K.: 1972, Solar Phys. 24, 87.

Bhattacharyya, J. C.: 1972, Solar Phys. 24, 274.

Bhavilai, R.: 1965, Monthly Notices Roy. Astron. Soc. 130, 411.

Bhavilai, R.: 1966, in K. O. Kiepenheuer (ed.), Fine Structure of the Solar Atmosphere, Franz Steiner Verlag, Wiesbaden, p. 96.

Bhavilai, R.: 1968, Solar Phys. 5, 471.

Bray, R. J.: 1968, Solar Phys. 5, 323.

Bray, R. J.: 1969, Solar Phys. 10, 63.

Bruzek, A.: 1959, Z. Astrophys. 47, 191.

Cram, L. E.: 1973, this symposium, p. 51.

de Jager, C. : 1957, Bull. Astron. Inst. Neth. 13, 133.

de Jager, C.: 1959, Handbuch der Physik 52, 80.

Dunn, R. B.: 1971, private communication.

Elliot, I.: 1969, Solar Phys. 6, 28.

Foukal, P.: 1971a, Solar Phys. 19, 59.

Foukal, P.: 1971b, Solar Phys. 20, 298.

Grossmann-Doerth, U. and von Uexküll, M.: 1971, Solar Phys. 20, 31.

Grossmann-Doerth, U. and von Uexküll, M.: 1973, Solar Phys. 28, 319.

Howard, R. and Harvey, J.: 1964, Astrophys. J. 139, 1328.

Janssens, T. J.: 1970, Solar Phys. 11, 222.

Leighton, R. B.: 1963, Ann. Rev. Astron. Astrophys. 1, 19.

Liu, S. Y.: 1972, Thesis, Maryland University.

Liu, S. Y.: 1973, Preprint (HAO).

Liu, S. Y. and Sheeley, N. R.: 1971, Solar Phys. 20, 282.

Liu, S. Y. and Smith, E. V. P.: 1972, Solar Phys. 24, 301.

Macris, C.: 1957, Ann. Astrophys. 5, 179.

Macris, C. and Alissandrakis, C. E.: 1970, Solar Phys. 11, 59.

Nakagawa, Y.: 1973, this symposium, p. 157.

Orrall, F. Q.: 1966, Astrophys. J. 143, 917.

Pasachoff, J. M.: 1970, Solar Phys. 12, 202.

Pasachoff, J. M., Noyes, R. W., and Beckers, J. M.: 1968, Solar Phys. 5, 131.

Rogers, E. H.: 1970, Solar Phys. 13, 57.

Sawyer, C.: 1972, Solar Phys. 24, 79.

Simon, G. and Leighton, R. B.: 1964, Astrophys. J. 140, 1120.

Suemoto, Z.: 1971, private communication.

Tanaka, K.: 1972, Report of Big Bear Solar Observatory, No. 125.

Wilson, P. R. and Evans, C. D.: 1971, Solar Phys. 18, 29.

Wilson, P. R., Rees, D. E., Beckers, J. M., and Brown, D. R.: 1972, Solar Phys. 25, 86.

\section{DISCUSSION}

Deubner: I would like to comment on the horizontal phase velocities of $100 \mathrm{~km} \mathrm{~s}^{-1}$ that you mentioned in your talk. It has been shown that these velocities are probably not real velocities and not even real phase velocities on the solar surface but can be explained by random phase relationships of individual elements, which are uncorrelated and pulsating on their own frequency and own phase.

Meyer: I would like to come back to the remark you made about the 180 -s oscillation in the chromosphere and about the question of whether these are propagating or not. I recall when some years ago Dr Schmidt and I investigated the oscillations of the atmosphere we found one particular mode that is a little bit curious. It is a surface wave at the interface between the corona and the chromosphere. Such a wave decreases in amplitude rapidly with increasing depth but stays in phase and has a relatively high frequency. I wonder if there are observations that would fit to this type of mode.

Tanaka: What is the frequency of that mode?

Meyer: The range near $180 \mathrm{~s}$ would be acceptable. Waves of this type would be seen most prominently in the upper chromosphere near the corona interface. Perhaps Stix has a comment.

Stix: I do not believe that the 180-s oscillations are surface gravity waves because the wavelength of such waves is too short to fit with Dr Tanaka's observations. 